\section{POSTTRAUMATIC STRESS DISORDER IS MORE COMMON IN CHILDHOOD CANCER SURVIVORS}

Posttraumatic stress disorder (PTSD) is commonly caused by exposure to a traumatic event that overwhelms an individual's coping abilities. Although the most common form of PTSD involves a violent act, there are other causes which physicians need to be aware of. One such cause is childhood cancer.

Margaret L. Stuber, MD, and colleagues, compared 6,542 childhood cancer survivors $>18$ years of age with 368 of their healthy siblings over a 16year period. Of these patients, $9 \%$ of cancer survivors and $2 \%$ of healthy siblings reported functional impairment as well as distress due to PTSD.

The cancer survivors' main symptoms were avoidance of reminders of their cancer diagnosis and treatment, being easily startled, being on edge, extreme anxiety, hypervigilance, increased arousal, and phobias. Survivors reported that these symptoms kept them from functioning as normal adults. PTSD was associated with being unmarried, unemployed, earning $<\$ 20,000 /$ year, and an education level of high school or lower.

According to Stuber and colleagues, the severity of cancer treatment in the 1970 s could be a contributing factor to the increase in PTSD rates observed in recent years. At that time, treatment consisted of head radiation for head tumors and more toxic pharmacotherapeutic options. It is thought that these treatments led to cognitive impairment, infertility, and stunted growth, which in turn has led to increased stress levels. The researchers believe that this cognitive impairment has hindered the ability of patients to both further their educations and attain quality employment that would provide them with adequate income. Today's treatment is less toxic and more improved supported care has been offered to these patients.

Stuber and colleagues believe that PTSD among childhood cancer survivors is highly treatable with medication and other therapeutic options. However, they note that addressing the issue will not be simple, and further research is needed to determine the proper treatment options for these patients.
Funding for this research was provided by the National Cancer Center. (Pediatrics. 2010;125(5)e1124-e1134.)—Christopher Naccari

\section{RESEARCHERS FIND rTMS EFFECTIVE IN TREATING TREATMENT-RESISTANT DEPRESSION}

There are a myriad of options available for the treatment of treatment-resistant depression (TRD). Repetitive transcranial magnetic stimulation (rTMS) has previously been shown to be effective in the treatment of TRD. However, researchers are concerned about this treatment option due to the dearth of quality research and mixed outcomes.

Mark S. George, MD, and colleagues, conducted a randomized clinical trial to gauge the efficacy of rTMS on TRD. They studied 190 patients who were being treated for depression but were not receiving pharmacotherapy. Of these patients, 92 received rTMS while 98 received a sham treatment.

Treatment consisted of rTMS being delivered to the left prefrontal cortex at $120 \%$ motor threshold $(10 \mathrm{~Hz}, 4$-sceond train duration, and 26 second intertrain interval) for $\mathbf{3 7 . 5}$ minutes via a figure-eight solid-core coil. Sham treatment consisted of a similar coil being placed in the same area, however, there was a metal insert blocking the magnetic field. Both groups received daily treatment for 3 weeks. Patients that improved were then blindly treated for another 3 weeks.

George and colleagues found that depression remitted in $14.1 \%$ of patients in the rTMS group and in $5.1 \%$ of the sham group. It should be noted that this study had an overall retention rate of $88 \%$ ( $86 \%$ rTMS group; $90 \%$ sham group). Overall, patients receiving rTMS had 4.2 greater odds $(95 \% \mathrm{Cl}, 1.32-13.24)$ of remitting their depression compared to the sham group. In an open-label follow-up, $30.2 \%$ of the rTMS group and $29.6 \%$ of the sham group remitted.

Funding for this research was provided by the National Institute of Mental Health as the Optimization of TMS for the Treatment of Depression Study. (Arch Gen Psychiatry. 2010;67:507-516).Christopher Naccari 


\section{BIPOLAR I MAINTENANCE TREATMENT}

GEOON + LTHIUM OR VALPROATE
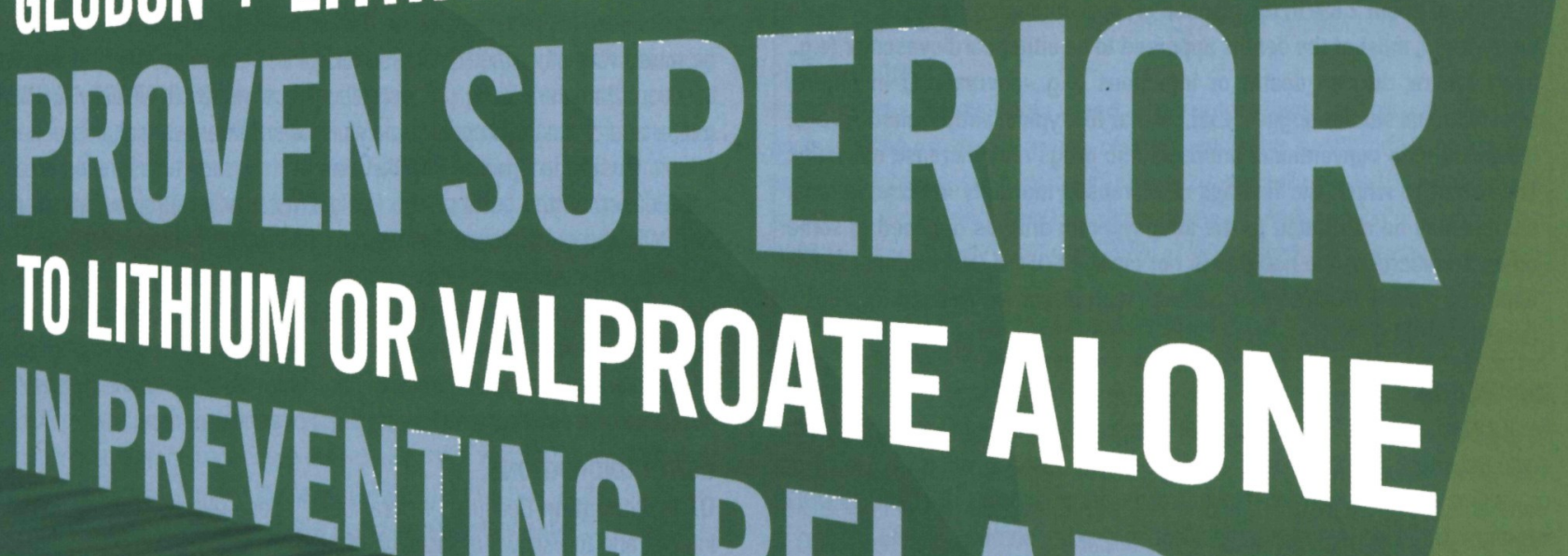


\section{GEODON ${ }^{\star}$ (ziprasidone HCl) Capsules}

\section{GEODON (ziprasidone mesylate) injection for intramuscular use}

BRIEF SUMMARY: See package insert for full prescribing information.

INCREASED MORTALITY IN ELDERLY PATIENTS WITH DEMENTIA-RELATED
PSYCHOSIS-Elderly patients with dementia-related pSychosis treated
with antipsychotic drugs are at an increased risk of death. Analyses of
seventeen placebo-controlled trials (modal duration of 10 weeks), largely
in patients taking atypical antipsychotic drugs, revealed a risk of death
in drug-treated patients of between 1.6 to 1.7 times the risk of death in
placebo-treated patients. Over the course of a typical 10-week controlled
trial, the rate of death in drug-treated patients was about 4.5\%, compared
to a rate of about $2.6 \%$ in the placebo group. Although the causes of death
were varied, most of the deaths appeared to be either cardiovascular (e.g.r
heart failure, sudden death) or infectious (e.g., pneumonia) in nature.
Observational studies suggest that, similar to atypical antipsychotic drugs,
treatment with conventional antipsychotic drugs may increase mortality.
The extent to which the findings of increased mortality in observational
studies may be attributed to the antipsychotic drug as opposed to some
characteristic(s) of the patients is not clear. GEODON (ziprasidone) is not
approved for the treatment of patients with Dementia-Related Psychosis
(see WARNINGS).

\section{INDICATIONS}

GEODON is indicated for the treatment of schizophrenia, as monotherapy for the acute treatment of bipolar manic or mixed episodes, and as an adjunct to lithium or valproate for the maintenance treatment of bipolar disorder. GEODON intramuscular is indicated for acute agitation in schizophrenic patients.

\section{DOSAGE AND ADMINISTRATION}

Schizophrenia GEODON Capsules should be administered at an initial daily dose of $20 \mathrm{mg}$ twice daily with food. In some patients, daily dosage may subsequently be adjusted on the basis of individual clinical status up to $80 \mathrm{mg}$ twice daily. Dosage adjustments, if indicated, should generally occur at intervals of not less than 2 days, as steady-state is achieved within 1 to 3 days. In order to ensure use of the lowest effective dose, patients should ordinarily be observed for improvement for several weeks before upward dosage adjustment. Efficacy in schizophrenia was demonstrated in a dose range of $20 \mathrm{mg}$ to $100 \mathrm{mg}$ twice daily in short-term, placebo-controlled clinical trials. There were trends toward dose response within the range of $20 \mathrm{mg}$ to $80 \mathrm{mg}$ twice daily, but results were not consistent. An increase to a dose greater than $80 \mathrm{mg}$ twice daily is not generally recommended. The safety of doses above $100 \mathrm{mg}$ twice daily has not been systematically evaluated in clinical trials. Maintenance Treatment-While there is no body of evidence available to answer the question of how long a patient treated with ziprasidone should remain on it, a maintenance study in patients who had been symptomatically stable and then randomized to continue ziprasidone or switch to placebo demonstrated a delay in time to relapse for patients receiving GEODON No additional benefit was demonstrated for doses above $20 \mathrm{mg}$ twice daily. Patients should be periodically reassessed to determine the need for maintenance treatment. Bipolar I Disorder Acute Treatment of Manic or Mixed EpisodesDose Selection: Oral ziprasidone should be administered at an initial daily dose of $40 \mathrm{mg}$ twice daily with food. The dose may then be increased to $60 \mathrm{mg}$ or $80 \mathrm{mg}$ twice daily on the second day of treatment and subsequently adjusted on the basis of tolerance and efficacy within the range $40 \mathrm{mg}$ to $80 \mathrm{mg}$ twice daily. In the flexible-dose clinical trials, the mean daily dose administered was approximately $120 \mathrm{mg}$. Maintenance Treatment (as an adjunct to lithium or valproate) - Continue treatment at the same dose on which the patient was initially stabilized, within the range of $40 \mathrm{mg}$ to $80 \mathrm{mg}$ twice daily with food. Patients should be periodically reassessed to determine the need for maintenance treatment. Acute Treatment of Agitation in Schizophrenia Intramuscular Dosing - The recommended dose is $10 \mathrm{mg}$ to $20 \mathrm{mg}$ administered as required up to a maximum dose of 40 $\mathrm{mg}$ per day. Doses of $10 \mathrm{mg}$ may be administered every two hours; doses of 20 $\mathrm{mg}$ may be administered every four hours up to a maximum of $40 \mathrm{mg} / \mathrm{day}$. Intramuscular administration of ziprasidone for more than three consecutive days has not been studied. If long-term therapy is indicated, oral ziprasidone hydrochloride capsules should replace the intramuscular administration as soon as possible. Since there is no experience regarding the safety of administering ziprasidone intramuscular to schizophrenic patients already taking oral ziprasidone, the practice of $\mathrm{co}$-administration is not recommended. Ziprasidone intramuscular is intended for intramuscular use only and should not be administered intravenously. intramuscular Preparation for Administration GEODON for Injection (ziprasidone mesylate) should only be administered by intramuscular injection and should not be administered intravenously. Single-dose vials require reconstitution prior to administration: Add $1.2 \mathrm{~mL}$ of Sterile Water for Injection to the vial and shake vigorously until all the drug is dissolved. Each $\mathrm{mL}$ of reconstituted solution contains $20 \mathrm{mg}$ ziprasidone. To administer a $10 \mathrm{mg}$ dose, draw up $0.5 \mathrm{~mL}$ of the reconstituted solution. To administer a $20 \mathrm{mg}$ dose, draw up $1.0 \mathrm{~mL}$ of the reconstituted solution. Any unused portion should be discarded. Since no preservative or bacteriostatic agent is present in this product, aseptic technique must be used in preparation of the final solution. This medicinal product must not be mixed with other medicinal products or solvents other than Sterile Water for Injection. Parenteral drug products should be inspected visually for particulate matter and discoloration prior to administration, whenever solution and container permit. Dosing in Special Populations Oral: Dosage adjustments are generally not required on the basis of age, gender, race, or renal or hepatic impairment. GEODON is not approved for use in children or adolescents. Intramuscular: Ziprasidone intramuscular has not been systematically evaluated in elderly patients or in patients with hepatic or renal impairment. As the cyciodextrin excipient is cleared by renal filtration, ziprasidone intramuscular should be administered with caution to patients with impaired renal function. Dosing adjustments are not required on the basis of gender or race.

\section{CONTRAINDICATIONS}

QT Prolongation Because of ziprasidone's dose-related prolongation of the QT interval and the known association of fatal arrhythmias with QT prolongation by some other drugs, ziprasidone is contraindicated in patients with a known history of QT prolongation (including congenital long QT syndrome), with recent acute myocardial infarction, or with uncompensated heart failure (see WARNINGS). Pharmacokinetic/pharmacodynamic studies between ziprasidone and other drugs that prolong the QT interval have not been performed. An additive effect of ziprasidone and other drugs that prolong the QT interval cannot be exciuded. Therefore, ziprasidone should not be given with dofetilide, sotalol, quinidine, other Class la and III anti-arrhythmics, mesoridazine, thioridazine, chlorpromazine, droperidol, pimozide, sparfloxacin, gatifloxacin, moxifloxacin, halofantrine, mefloquine, pentamidine, arsenic trioxide, levomethadyl acetate, dolasetron mesylate, probucol or tacrolimus. Ziprasidone is also contraindicated with other drugs that have demonstrated QT prolongation as one of their pharmacodynamic effects and have this effect described in the full prescribing information as a contraindication or a boxed or bolded warning [see WARNINGS]. Ziprasidone is contraindicated in individuals with a known hypersensitivity to the product.

\section{WARNINGS}

Increased Mortality in Elderly Patients with Dementia-Related Psychosis: Elderly patients with dementia-related psychosis treated with antipsychotic drugs are at an increased risk of death. GEODON is not approved for the treatment of dementia-related psychosis (see BOXED WARNING).

QT Prolongation and Risk of Sudden Death Ziprasidone use should be avoided in combination with other drugs that are known to prolong the QTc interval. Additionally, clinicians should be alert to the identification of other drugs that have been consistently observed to prolong the $Q T_{c}$ interval. Such drugs should not be prescribed with ziprasidone. Ziprasidone should also be avoided in patients with congenital long QT syndrome and in patients with a history of cardiac arrhythmias (see CONTRAINDICATIONS).

QT Prolongation in Clinical Trials A study directly comparing the QT/QTC prolonging effect of oral ziprasidone with several other drugs effective in the treatment of schizophrenia was conducted in patient volunteers. The mean increase in $Q T_{c}$ from baseline for ziprasidone ranged from approximately 9 to 14 msec greater than for four of the comparator drugs (risperidone, olanzapine, quetiapine, and haloperidol), but was approximately $14 \mathrm{msec}$ less than the prolongation observed for thioridazine. In this study, the effect of ziprasidone on QTc length was not augmented by the presence of a metabolic inhibitor (ketoconazole 
$200 \mathrm{mg}$ twice daily). In placebo-controlled trials, oral ziprasidone increased the $Q T_{c}$ interval compared to placebo by approximately $10 \mathrm{msec}$ at the highest recommended daily dose of $160 \mathrm{mg}$. In clinical trials the electrocardiograms of $2 / 2988(0.06 \%)$ patients who received GEODON and $1 / 440(0.23 \%)$ patients who received placebo revealed $Q T_{c}$ intervals exceeding the potentially clinically relevant threshold of $500 \mathrm{msec}$. In the ziprasidone-treated patients, neither case suggested a role of ziprasidone. QT Prolongation and Torsade De Pointes Some drugs that prolong the $Q T / Q T_{c}$ interval have been associated with the occurrence of torsade de pointes and with sudden unexplained death. The relationship of QT prolongation to torsade de pointes is clearest for larger increases $(20 \mathrm{msec}$ and greater) but it is possible that smaller $Q T / Q T_{c}$ prolongations may also increase risk, or increase it in susceptible individuals. Although torsade de pointes has not been observed in association with the use of ziprasidone in premarketing studies and experience is too limited to rule out an increased risk, there have been rare post-marketing reports (in the presence of multiple confounding factors) (see ADVERSE REACTIONS). A study evaluating the QT/QTc prolonging effect of intramuscular ziprasidone, with intramuscular haloperidol as a control, was conducted in patient volunteers. In the trial, ECGs were obtained at the time of maximum plasma concentration following two injections of ziprasidone $(20 \mathrm{mg}$ then $30 \mathrm{mg}$ ) or haloperidol ( $7.5 \mathrm{mg}$ then $10 \mathrm{mg}$ ) given four hours apart. Note that a $30 \mathrm{mg}$ dose of intramuscular ziprasidone is $50 \%$ higher than the recommended therapeutic dose. The mean change in $\mathrm{QT}_{\mathrm{c}}$ from baseline was calculated for each drug, using a sample-based correction that removes the effect of heart rate on the QT interval. The mean increase in $Q T_{c}$ from baseline for ziprasidone was 4.6 msec following the first injection and $12.8 \mathrm{msec}$ following the second injection. The mean increase in QTc from baseline for haloperidol was $6.0 \mathrm{msec}$ following the first injection and $14.7 \mathrm{msec}$ following the second injection. In this study, no patients had a $Q T_{c}$ interval exceeding $500 \mathrm{msec}$. As with other antipsychotic drugs and placebo, sudden unexplained deaths have been reported in patients taking ziprasidone at recommended doses. The premarketing experience for ziprasidone did not reveal an excess risk of mortality for ziprasidone compared to other antipsychotic drugs or placebo, but the extent of exposure was limited, especially for the drugs used as active controls and placebo. Nevertheless, ziprasidone's larger prolongation of $\mathrm{QT}_{\mathrm{c}}$ length compared to several other antipsychotic drugs raises the possibility that the risk of sudden death may be greater for ziprasidone than for other available drugs for treating schizophrenia. This possibility needs to be considered in deciding among alternative drug products. Certain circumstances may increase the risk of the occurrence of torsade de pointes and/or sudden death in association with the use of drugs that prolong the QTC interval, including (1) bradycardia; (2) hypokalemia or hypomagnesemia; (3) concomitant use of other drugs that prolong the QTc interval; and (4) presence of congenital prolongation of the QT interval. Electrolyte Disturbances May Increase The Risk of QT Prolongation it is recommended that patients being considered for ziprasidone treatment who are at risk for significant electrolyte disturbances, hypokalemia in particular, have baseline serum potassium and magnesium measurements. Hypokalemia (and/or hypomagnesemia) may increase the risk of QT prolongation and arrhythmia. Hypokalemia may result from diuretic therapy, diarrhea, and other causes. Patients with low serum potassium and/or magnesium should be repleted with those electrolytes before proceeding with treatment. It is essential to periodically monitor serum electrolytes in patients for whom diuretic therapy is introduced during ziprasidone treatment. Persistently prolonged $\mathrm{QT}_{\mathrm{c}}$ intervals may also increase the risk of further prolongation and arrhythmia, but it is not clear that routine screening ECG measures are effective in detecting such patients. Rather, ziprasidone should be avoided in patients with histories of significant cardiovascular illness, e.g., QT prolongation, recent acute myocardial infarction, uncompensated heart failure, or cardiac arrhythmia. Ziprasidone should be discontinued in patients who are found to have persistent $Q T_{c}$ measurements $>500 \mathrm{msec}$. Neuroleptic Malignant Syndrome (NMS) A potentially fatal symptom complex sometimes referred to as Neuroleptic Malignant Syndrome (NMS) has been reported in association with administration of antipsychotic drugs. The management of NMS should include: (1) immediate discontinuation of antipsychotic drugs and other drugs not essential to concurrent therapy; (2) intensive symptomatic treatment and medical monitoring; and (3) treatment of any concomitant serious medical problems for which specific treatments are available. If a patient requires antipsychotic drug treatment after recovery from NMS, the potential reintroduction of drug therapy should be carefully considered. The patient should be carefully monitored, since recurrences of NMS have been reported. Tardive Dyskinesia A syndrome of potentially irreversible, involuntary, dyskinetic movements may develop in patients undergoing treatment with antipsychotic drugs. Although the prevalence of the syndrome appears to be highest among the elderly, especially elderly women, it is impossible to rely upon prevalence estimates to predict, at the inception of antipsychotic treatment, which patients are likely to develop the syndrome. If signs and symptoms of tardive dyskinesia appear in a patient on ziprasidone, drug discontinuation should be considered. Hyperglycemia and Diabetes Mellitus Hyperglycemia-related adverse events, sometimes serious, have been reported in patients treated with atypical anti-psychotics. There have been few reports of hyperglycemia or diabetes in patients treated with GEODON, and it is not known if GEODON is associated with these events. Any patient treated with atypical antipsychotics should be monitored for symptoms of hyperglycemia.

\section{PRECAUTIONS}

Leukopenia, Neutropenia, and Agranulocytosis In clinical trial and postmarketing experience, events of leukopenia/neutropenia and agranulocytosis (including fatal cases) have been reported temporally related to antipsychotic agents. Possible risk factors for leukopenia/neutropenia include pre-existing low white blood cell count (WBC) and history of drug induced leukopenia/neutropenia. Patients with a pre-existing low WBC or a history of drug induced leukopenia/ neutropenia should have their complete blood count (CBC) monitored frequently during the first few months of therapy and should discontinue GEODON at the first sign of decline in WBC in the absence of other causative factors. Patients with neutropenia should be carefully monitored for fever or other symptoms or signs of infection and treated promptly if such symptoms or signs occur. Patients with severe neutropenia (absolute neutrophil count $<1000 / \mathrm{mm} 3$ ) should discontinue GEODON and have their WBC followed until recovery. Rash In premarketing trials with ziprasidone, about $5 \%$ of patients developed rash and/or urticaria, with discontinuation of treatment in about one-sixth of these cases. The occurrence of rash was related to dose of ziprasidone, although the finding might also be explained by the longer exposure time in the higher dose patients. Several patients with rash had signs and symptoms of associated systemic illness, e.g., elevated WBCs. Most patients improved promptly with adjunctive treatment with antihistamines or steroids and/or upon discontinuation of ziprasidone, and all patients experiencing these reactions were reported to recover completely. Upon appearance of rash for which an alternative etiology cannot be identified, ziprasidone should be discontinued. Orthostatic Hypotension Ziprasidone may induce orthostatic hypotension associated with dizziness, tachycardia, and, in some patients, syncope, especially during the initial dose-titration period, probably reflecting its $\alpha$-adrenergic antagonist properties. Syncope was reported in $0.6 \%$ of the patients treated with ziprasidone. Ziprasidone should be used with particular caution in patients with known cardiovascular disease (history of myocardial infarction or ischemic heart disease, heart failure or conduction abnormalities), cerebrovascular disease, or conditions which would predispose patients to hypotension (dehydration, hypovolemia, and treatment with antihypertensive medications). Seizures In clinical trials, seizures occurred in $0.4 \%$ of patients treated with ziprasidone. There were confounding factors that may have contributed to the occurrence of seizures in many of these cases. As with other antipsychotic drugs, ziprasidone should be used cautiously in patients with a history of seizures or with conditions that potentially lower the seizure threshold, e.g., Alzheimer's dementia. Conditions that lower the seizure threshold may be more prevalent in a population of 65 years or older. Dysphagia Esophageal dysmotility and aspiration have been associated with antipsychotic drug use. Aspiration pneumonia is a common cause of morbidity and mortality in elderly patients, in particular those with advanced Alzheimer's dementia, and ziprasidone and other antipsychotic drugs should be used cautiously in patients at risk for aspiration pneumonia (see BOXED WARNING and Increased Mortality in Elderly Patients with Dementia-Related Psychosis in WARNINGS). Hyperprolactinemia As with other drugs that antagonize dopamine D2 receptors, ziprasidone elevates prolactin levels in humans. Tissue culture experiments indicate that approximately one-third of human breast cancers are prolactin-dependent in vitro, a factor of potential importance if the prescription of these drugs is contemplated in a patient with previously detected breast cancer. Neither clinical studies nor epidemiologic studies conducted to date have shown an association between chronic 
administration of this class of drugs and tumorigenesis in humans; the available evidence is considered too limited to be conclusive at this time. Potential for Cognitive and Motor Impairment Somnolence was a commonly reported adverse reaction in patients treated with ziprasidone. In the 4- and 6-week placebo-controlled trials, somnolence was reported in $14 \%$ of patients on ziprasidone compared to $7 \%$ of placebo patients. Somnolence led to discontinuation in $0.3 \%$ of patients in short-term clinical trials. Since ziprasidone has the potential to impair judgment, thinking, or motor skills, patients should be cautioned about performing activities requiring mental alertness, such as operating a motor vehicle (inc/uding automobiles) or operating hazardous machinery until they are reasonably certain that ziprasidone therapy does not affect them adversely. Priapism One case of priapism was reported in the premarketing database. Body Temperature Regulation Although not reported with ziprasidone in premarketing trials, disruption of the body's ability to reduce core body temperature has been attributed to antipsychotic agents. Suicide The possibility of a suicide attempt is inherent in psychotic illness and close supervision of high-risk patients should accompany drug therapy. Prescriptions for ziprasidone should be written for the smallest quantity of capsules consistent with good patient management in order to reduce overdose risk. Patients With Concomitant IIInesses Clinical experience with ziprasidone in patients with certain concomitant systemic illnesses is limited. Ziprasidone has not been evaluated or used to any appreciable extent in patients with a recent history of myocardial infarction or unstable heart disease. Patients with these diagnoses were excluded from premarketing clinical studies. Because of the risk of QTc prolongation and orthostatic hypotension with ziprasidone, caution should be observed in cardiac patients (see QT Prolongation and Risk of Sudden Death in WARNINGS and Orthostatic Hypotension in PRECAUTIONS). Information for Patients To assure safe and effective use of GEODON, the information and instructions provided in the patient information should be discussed with patients. Laboratory Tests Patients being considered for ziprasidone treatment who are at risk of significant electrolyte disturbances should have baseline serum potassium and magnesium measurements. Low serum potassium and magnesium should be replaced before proceeding with treatment. Patients who are started on diuretics during Ziprasidone therapy need periodic monitoring of serum potassium and magnesium. Discontinue ziprasidone in patients who are found to have persistent $Q T_{c}$ measurements $>500 \mathrm{msec}$ (see WARNINGS).

\section{DRUG INTERACTIONS}

(1) Ziprasidone should not be used with any drug that prolongs the QT interval. (2) Given the primary CNS effects of ziprasidone, caution should be used when it is taken in combination with other centrally acting drugs. (3) Because of its potential for inducing hypotension, ziprasidone may enhance the effects of certain antihypertensive agents. (4) Ziprasidone may antagonize the effects of levodopa and dopamine agonists. Effect of Other Drugs on Ziprasidone Carbamazepine, $200 \mathrm{mg}$ bid for 21 days, resulted in a decrease of approximately $35 \%$ in the AUC of ziprasidone. Ketoconazole, a potent inhibitor of CYP $3 A 4,400 \mathrm{mg}$ qd for 5 days, increased the AUC and Cmax of ziprasidone by about $35-40 \%$. Cimetidine, 800 $\mathrm{mg}$ qd for 2 days, did not affect ziprasidone pharmacokinetics. Co-administration of $30 \mathrm{~mL}$ of Maalox® did not affect ziprasidone pharmacokinetics. Population pharmacokinetic analysis of schizophrenic patients enrolled in controlled clinical trials has not revealed evidence of any clinically significant pharmacokinetic interactions with benztropine, propranolol, or lorazepam. Effect of Ziprasidone on Other Drugs in vitro studies revealed little potential for ziprasidone to interfere with the metabolism of drugs cleared primarily by CYP1A2, CYP2C9, CYP2C19, CYP2D6 and CYP3A4, and little potential for drug interactions with ziprasidone due to displacement. Ziprasidone $40 \mathrm{mg}$ bid administered concomitantly with lithium $450 \mathrm{mg}$ bid for 7 days did not affect the steady-state level or renal clearance of lithium. In vivo studies have revealed no effect of ziprasidone on the pharmacokinetics of estrogen or progesterone components. Ziprasidone $20 \mathrm{mg}$ bid did not affect the pharmacokinetics of concomitantly administered oral contraceptives, ethinyl estradiol $(0.03 \mathrm{mg})$ and levonorgestrel $(0.15 \mathrm{mg})$. Consistent with in vitro results, a study in normal healthy volunteers showed that ziprasidone did not alter the metabolism of dextromethorphan, a CYP2D6 model substrate, to its major metabolite, dextrorphan. There was no statistically significant change in the urinary dextromethorphan/dextrorphan ratio.

\section{NONCLINICAL TOXICOLOGY}

Carcinogenesis, Mutagenesis, Impairment of Fertility Lifetime carcinogenicity studies were conducted with ziprasidone in Long Evans rats and CD-1 mice. In male mice, there was no increase in incidence of tumors relative to controls. In female mice, there were dose-related increases in the incidences of pituitary gland adenoma and carcinoma, and mammary gland adenocarcinoma at all doses tested. Increases in serum prolactin were observed in a 1-month dietary study in female, but not male, mice. Ziprasidone had no effect on serum prolactin in rats in a 5-week dietary study at the doses that were used in the carcinogenicity study. The relevance for human risk of the findings of prolactin-mediated endocrine tumors in rodents is unknown (see Hyperprolactinemia in PRECAUTIONS). Mutagenesis: There was a reproducible mutagenic response in the Ames assay in one strain of $S$. typhimurium in the absence of metabolic activation. Positive results were obtained in both the in vitro mammalian cell gene mutation assay and the in vitro chromosomal aberration assay in human lymphocytes. Impairment of Fertility: Ziprasidone increase time to copulation in Sprague-Dawley rats in two fertility and early embryonic development studies at doses of 10 to $160 \mathrm{mg} / \mathrm{kg} /$ day ( 0.5 to 8 times the MRHD of $200 \mathrm{mg} /$ day on a $\mathrm{mg} / \mathrm{m} 2$ basis). Fertility rate was reduced at $160 \mathrm{mg} / \mathrm{kg} /$ day ( 8 times the MRHD on a $\mathrm{mg} / \mathrm{m} 2$ basis). There was no effect on fertility at $40 \mathrm{mg} / \mathrm{kg} /$ day (2 times the MRHD on a $\mathrm{mg} / \mathrm{m} 2$ basis). The fertility of female rats was reduced.

\section{USE IN SPECIFIC POPULATIONS}

Pregnancy Pregnancy Category C: There are no adequate and well-controlled studies in pregnant women. Ziprasidone should be used during pregnancy only if the potential benefit justifies the potential risk to the fetus. Labor and Delivery The effect of ziprasidone on labor and delivery in humans is unknown. Nursing Mothers It is not known whether ziprasidone or its metabolites are excreted in human milk. It is recommended that women receiving ziprasidone should not breastfeed. Pediatric Use The safety and effectiveness of ziprasidone in pediatric patients have not been established. Geriatric Use of the total number of subjects in clinical studies of ziprasidone, 2.4 percent were 65 and over. No overall differences in safety or effectiveness were observed between these subjects and younger subjects. Nevertheless, the presence of multiple factors that might increase the pharmacodynamic response to ziprasidone, or cause poorer tolerance or orthostasis, should lead to consideration of a lower starting dose, slower titration, and careful monitoring during the initial dosing period for some elderly patients.

\section{ADVERSE REACTIONS}

Adverse Findings Observed in Short-term, Placebo-Controlled Trials The following findings are based on the short-term placebo-controlled premarketing trials for schizophrenia (a pool of two 6-week, and two 4-week fixed-dose trials) and bipolar mania (a pool of two 3-week flexible-dose trials) in which GEODON was administered in doses ranging from 10 to $200 \mathrm{mg} /$ day. Adverse Events Associated With Discontinuation Schizophrenia: Approximately $4.1 \%$ (29/702) of ziprasidone-treated patients in short-term, placebo-controlled studies discontinued treatment due to an adverse reaction, compared with about $2.2 \%$ (6/273) on placebo. The most common reaction associated with dropout was rash, including 7 dropouts for rash among ziprasidone patients (1\%) compared to no placebo patients (see PRECAUTIONS). Bipolar Mania: Approximately $6.5 \%$ (18/279) of ziprasidone-treated patients in short-term, placebo-controlled studies discontinued treatment due to an adverse reaction, compared with about $3.7 \%$ (5/136) on placebo. The most common reactions associated with dropout in the ziprasidone-treated patients were akathisia, anxiety, depression, dizziness, dystonia, rash and vomiting, with 2 dropouts for each of these reactions among ziprasidone patients (1\%) compared to one placebo patient each for dystonia and rash (1\%) and no placebo patients for the remaining adverse events. Adverse Events at an Incidence of $\geq 5 \%$ and at Least Twice the Rate of Placebo The most commonly observed adverse events associated with GEODON in schizophrenia trials were somnolence $(14 \%)$ and respiratory tract infection $(8 \%)$. The most commonly observed adverse events associated with the use of GEODON in bipolar mania trials were somnolence (31\%), extrapyramidal symptoms $(31 \%)$, dizziness $(16 \%)$, akathisia $(10 \%)$, abnormal vision $(6 \%)$, asthenia $(6 \%)$, and vomiting $(5 \%)$. The following list enumerates the treatment-emergent adverse events that occurred during acute therapy, including only those events that 
occurred in $2 \%$ of GiEUUUN patients and at a greater incidence than in placebo. Schizophrenia: Body as a Whole-asthenia, accidental injury, chest pain. Cardiovascular-tachycardia. Digestive-nausea, constipation, dyspepsia, diarrhea, dry mouth, anorexia. Nervous - extrapyramidal symptoms, somnolence, akathisia, dizziness. Respiratory—respiratory 'tract infection, rhinitis, cough increased. Skin and Appendages-rash, fungal dermatitis. Special Sensesabnormal vision. Bipolar Mania: Body as a Whole - headache, asthenia, accidental injury. Cardiovascular-hypertension. Digestive - nausea, diarrhea, dry mouth, vomiting, increased salivation, tongue edema, dysphagia. Musculoskeletalmyalgia. Nervous - somnolence, extrapyramidal symptoms, dizziness, akathisia, anxiety, hypesthesia, speech disorder. Respiratory—pharyngitis, dyspnea. Skin and Appendages - fungal dermatitis. Special Senses-abnormal vision. Dose Dependency An analysis for dose response in the schizophrenia 4-study pool revealed an apparent relation of adverse reaction to dose for the following reactions: asthenia, postural hypotension, anorexia, dry mouth, increased salivation, arthralgia, anxiety, dizziness, dystonia, hypertonia, somnolence, tremor, rhinitis, rash, and abnormal vision. Extrapyramidal Symptoms (EPS) The incidence of reported EPS for ziprasidone patients in the short-term, placebocontrolled schizophrenia trials was $14 \%$ vs. $8 \%$ for placebo. Objectively collected data from those trials on the Simpson-Angus Rating Scale (for EPS) and the Barnes Akathisia Scale (for akathisia) did not generally show a difference between ziprasidone and placebo. Dystonia Symptoms of dystonia, prolonged abnormal contractions of muscle groups, may occur in susceptible individuals during the first few days of treatment. While these symptoms can occur at low doses, they occur more frequently and with greater severity with high potency and at higher doses of first generation antipsychotic drugs. Elevated risk of acute dystonia is observed in males and younger age groups. Vital Sign Changes Ziprasidone is associated with orthostatic hypotension (see PRECAUTIONS). Weight Gain in short-term schizophrenia trials, the proportions of patients meeting a weight gain criterion of $\geq 7 \%$ of body weight were compared, revealing a statistically significantly greater incidence of weight gain for ziprasidone (10\%) compared to placebo (4\%). A median weight gain of $0.5 \mathrm{~kg}$ was observed in ziprasidone patients compared to no median weight change in placebo patients. Weight gain was reported as an adverse event in $0.4 \%$ of both ziprasidone and placebo patients. During long-term therapy with ziprasidone, a categorization of patients at baseline on the basis of body mass index (BMI) revealed the greatest mean weight gain and highest incidence of clinically significant weight gain $(>7 \%$ of body weight) in patients with low BMI $(<23)$ compared to normal $(23-27)$ or overweight patients (>27). There was a mean weight gain of $1.4 \mathrm{~kg}$ for those patients with a "low" baseline BMI, no mean change for patients with a "normal" BMl, and a $1.3 \mathrm{~kg}$ mean weight loss for patients who entered the program with a "high" BMl. ECG Changes Ziprasidone is associated with an increase in the $Q T_{c}$ interval (see WARNINGS). In the schizophrenia trials, ziprasidone was associated with a mean increase in heart rate of 1.4 beats per minute compared to a 0.2 beats per minute decrease among placebo patients. Other Adverse Events Observed During the Premarketing Evaluation of Ziprasidone in Schizophrenia Frequent adverse events are those occurring in at least $1 / 100$ patients; infrequent adverse events are those occurring in 1/100 to $1 / 1000$ patients; rare adverse events are those occurring in fewer than $1 / 1000$ patients. Body as a Whole-Frequent abdominal pain, flu syndrome, fever, accidental fall, face edema, chills photosensitivity reaction, flank pain, hypothermia, motor vehicle accident. Cardiovascular System-Frequent: tachycardia, hypertension, postural hypotension. Infrequent: bradycardia, angina pectoris, atrial fibrillation. Rare: first degree AV block, bundle branch block, phlebitis, pulmonary embolus, cardiomegaly, cerebral infarct, cerebrovascular accident, deep thrombophlebitis, myocarditis, thrombophlebitis. Digestive System-Frequent: anorexia, vomiting. Infrequent rectal hemorrhage, dysphagia, tongue edema. Rare: gum hemorrhage, jaundice, fecal impaction, gamma glutamyl trans-peptidase increased, hematemesis, cholestatic jaundice, hepatitis, hepatomegaly, leukoplakia of mouth, fatty liver deposit, melena. Endocrine-Rare: hypothyroidism, hyperthyroidism, thyroiditis. Hemic and Lymphatic System — Infrequent: anemia, ecchymosis, leukocytosis, leukopenia, eosinophilia, lymphadenopathy. Rare: thrombocytopenia, hypochromic anemia, lymphocytosis, monocytosis, basophilia, lymphedema, polycythemia, thrombocythemia. Metabolic and Nutritional Disorders-Infrequent: thirst, transaminase increased, peripheral edema, hyperglycemia, creatine phosphokinase

Increased, alkaline pnosphatase increased, hypercholesteremı, dehydration, lactic dehydrogenase increased, albuminuria, hypokalemia. Rare: BUN increased, creatinine increased, hyperlipemia, hypocholesteremia, hyperkalemia, hypochloremia, hypoglycemia, hyponatremia, hypoproteinemia, glucose tolerance decreased, gout, hyperchloremia, hyperuricemia, hypocalcemia, hypoglycemic reaction, hypomagnesemia, ketosis, respiratory alkalosis. Musculoskeletal System - Frequent: myalgia. Infrequent: tenosynovitis. Rare: myopathy. Nervous System - Frequent: agitation, extrapyramidal syndrome, tremor, dystonia, hypertonia, dyskinesia, hostility, twitching, paresthesia, confusion, vertigo, hypokinesia, hyperkinesia, abnormal gait, oculogyric crisis, hypesthesia, ataxia, amnesia, cogwheel rigidity, delirium, hypotonia, akinesia, dysarthria, withdrawal syndrome, buccoglossal syndrome, choreoathetosis, diplopia, incoordination, neuropathy. Infrequent: paralysis. Rare: myoclonus, nystagmus, torticollis, circumoral paresthesia, opisthotonos, reflexes increased, trismus. Respiratory System-Frequent: dyspnea Infrequent pneumonia, epistaxis. Rare: hemoptysis, laryngismus. Skin and Appendages—infrequent: maculopapular rash, urticaria, alopecia, eczema, exfoliative dermatitis, contact dermatitis, vesiculobullous rash. Special Senses-Frequent: fungal dermatitis. Infrequent: conjunctivitis, dry eyes, tinnitus, blepharitis, cataract, photophobia. Rare: eye hemorrhage, visual field defect, keratitis, keratoconjunctivitis. Urogenital System-Infrequent: impotence, abnormal ejaculation, amenorrhea, hematuria, menorrhagia, female lactation, polyuria, urinary retention, metrorrhagia, male sexual dysfunction, anorgasmia, glycosuria. Rare: gynecomastia, vaginal hemorrhage, nocturia, oliguria, female sexual dysfunction, uterine hemorrhage. Adverse Findings Observed in Trials of Intramuscular Ziprasidone In these studies, the most commonly observed adverse reactions associated with the use of intramuscular ziprasidone $(\geq 5 \%)$ and observed at a rate on intramuscular ziprasidone (in the higher dose groups) at least twice that of the lowest intramuscular ziprasidone group were headache $(13 \%)$, nausea (12\%), and somnolence (20\%). Adverse Events at an Incidence of $\geq 1 \%$ in Short-Term Fixed-Dose Intramuscular Trials The following list enumerates the treatment-emergent adverse events that occurred in $\geq 1 \%$ of patients during acute therapy with intramuscular ziprasidone: Body as a Wholeheadache, injection site pain, asthenia, abdominal pain, flu syndrome, back pain. Cardiovascular-postural hypotension, hypertension, bradycardia, vasodilation. Digestive-nausea, rectal hemorrhage, diarrhea, vomiting, dyspepsia, anorexia, constipation, tooth disorder, dry mouth. Nervous-dizziness, anxiety, insomnia, somnolence, akathisia, agitation, extrapyramidal syndrome, hypertonia, cogwheel rigidity, paresthesia, personality disorder, psychosis, speech disorder. Respiratoryrhinitis. Skin and Appendages-furunculosis, sweating. Urogenitadysmenorrhea, priapism. Other Events Observed During Post-marketing Use Adverse reaction reports not listed above that have been received since market introduction include rare occurrences of the following-Cardiac Disorders: Tachycardia, torsade de pointes (in the presence of multiple confounding factors), (see WARNINGS); Digestive System Disorders: Swollen Tongue; Reproductive System and Breast Disorders: Galactorrhea, priapism; Nervous System Disorders: Facial Droop, neuroleptic malignant syndrome, serotonin syndrome (alone or in combination with serotonergic medicinal products), tardive dyskinesia; Psychiatric Disorders: Insomnia, mania/hypomania; Skin and subcutaneous Tissue Disorders: Allergic reaction (such as allergic dermatitis, angioedema, orofacial edema, urticaria), rash; Urogenital System Disorders: Enuresis, urinary incontinence; Vascular Disorders: Postural hypotension, syncope.

\section{DRUG ABUSE AND DEPENDENCE \\ Controlled Substance Class Ziprasidone is not a controlled substance.}

\section{OVERDOSAGE}

In premarketing trials in over 5400 patients, accidental or intentional overdosage of oral ziprasidone was documented in 10 patients. All patients survived without sequelae. In the patient taking the largest confirmed amount $(3240 \mathrm{mg}$ ), the only symptoms reported were minimal sedation, slurring of speech, and transitory hypertension (200/95).

(n)

